\title{
Étude Ethnobotanique Des Plantes Utilisées Dans L'artisanat Chez Les Agni Du Centre-Est Et Nord-Est De La Côte d'Ivoire
}

\author{
Amani Léopold Litta \\ Amadou Lamine Diop \\ Yao Bertin Kouakou
}

U.F.R. des Sciences de la Nature, Abidjan, Côte d'Ivoire

Djah François Malan

Université Nangui Abrogoua,

U.F.R. des Sciences de la Nature, Abidjan, Côte d'Ivoire

Institut Botanique Aké-Assi d'Andokoi, Abidjan, Côte d'Ivoire

\section{Doi:10.19044/esj.2021.v17n3p133}

Submitted: 18 December 2020

Accepted: 27 October 2020

Published: 31 October 2020
Copyright 2021 Author(s)

Under Creative Commons BY-NC-ND

4.0 OPEN ACCESS

Cite As:

Léopold Litta A., Lamine Diop A., Bertin Kouakou Y. \& François Malan D. (2021). Étude Ethnobotanique Des Plantes Utilisées Dans L'artisanat Chez Les Agni Du Centre-Est Et Nord-Est De La Côte d'Ivoire. European Scientific Journal, ESJ, 17(3),133.

https://doi.org/10.19044/esj.2021.v17n3p133

\section{Résumé}

Dans le but de valoriser le savoir traditionnel des plantes utilisées dans l'artisanat, une enquête ethnobotanique a été conduite chez quatre tribus Agni du Centre-est et du Nord-est de la Côte d'Ivoire. Les entretiens semi-structurés à travers l'approche du « porte-à-porte » suivis de randonnées dans le milieu environnant ont permis d'obtenir les informations sur l'usage des plantes. Cette étude a montré que 104 espèces réparties en 95 genres et 35 familles sont utilisées dans l'artisanat. Pour ces quatre tribus, seulement 14,42\% des plantes ont un indice d'importance culturelle élevé. Il s'agit entre autres de Baissea multiflora, Holarrhena floribunda, Mansonia altissima, Nauclea diderrichii, Nesogordonia papaverifera, Pseudocedrela kotschyi, etc. L'analyse du coefficient de Jaccard a montré des différences majeures au niveau de l'usage des plantes entre ces quatre communautés, avec seulement 13,46\% d'usage commun. Il a été également observé que les savoirs liés aux plantes à usage artisanal s'érodent progressivement, à cause notamment de l'abondance des 
matériaux modernes et surtout de la raréfaction des plantes utilisées due à la dégradation de l'environnement.

Mots-clés: Plantes Artisanales, Tribus Agni, Agni-Abè, Agni-Barabo, AgniBini, Agni-Bona, Côte d'Ivoire

\title{
Ethnobotanical Study Of Plants Used In Handicrafts, And Comparison Of Traditional Knowledge: The Case Of The Agni Communities Of Central-Eastern And North-Eastern Côte d'Ivoire
}

\section{Amani Léopold Litta \\ Amadou Lamine Diop \\ Yao Bertin Kouakou}

U.F.R. des Sciences de la Nature, Abidjan, Côte d'Ivoire

\section{Djah François Malan}

Université Nangui Abrogoua,

U.F.R. des Sciences de la Nature, Abidjan, Côte d'Ivoire Institut Botanique Aké-Assi d'Andokoi, Abidjan, Côte d'Ivoire

\begin{abstract}
An ethnobotanical survey was conducted among four Agni tribes in the central-eastern and north-eastern parts of Côte d'Ivoire. This study aimed to valorize the traditional knowledge of plants used in handicrafts. Semistructured interviews through the "door-to-door" approach followed by walks in the adjacent vegetation provided information on the use of plants. This study showed that 104 species divided into 95 genera and 35 families are used in local handicrafts. For these four tribes, only $14.42 \%$ of the plants have a high index of cultural importance. These include Baissea multiflora, Holarrhena floribunda, Mansonia altissima, Nauclea diderrichii, Nesogordonia papaverifera, Pseudocedrela kotschyi etc. The analysis of Jaccard's coefficient showed differences in the use of plants between these four communities, with only $13.46 \%$ of these plants in common use. The knowledge related to plants for artisanal use is gradually deteriorating, for several reasons: obsolescence of uses, the unavailability of plants due to environmental degradation and competition with modern materials.
\end{abstract}

Keywords: Artisanal Plants, Agni Tribes, Agni-Abè, Agni-Barabo, AgniBini, Agni-Bona, Côte d'Ivoire

Introduction 
En milieu rural, les populations ont des connaissances traditionnelles des plantes, du fait de leur utilisation ancestrale (Ndiaye et al., 2017). De nombreuses plantes sont utilisées par différents peuples pour construire des habitats traditionnels et pour fabriquer différents outils ou articles utilisés au quotidien (Kouakou et al., 2020). Ces plantes utilisées dans l'artisanat d'art ou utilitaire occupent une place importante dans le bien-être social des populations rurales. En effet, Elles permettent d'avoir des habitats à moindre coût pour s'abriter, des ustensiles et outils nécessaires pour la vie quotidienne des populations attachées à leur tradition et/ou par manque de ressources financières (Tiébré et al., 2016). L'artisanat contribue également au rayonnement culturel d'un peuple. Ce domaine informel met en action des acteurs faisant preuve d'une créativité remarquable reposant sur des savoirs locaux détenus essentiellement par certains groupes dans la population (PNUD, 2006). Elle est une source de revenus notable pour ces populations (Tiébré et al., 2016). Par conséquent, la surexploitation et la déforestation due à la pression anthropique sont un risque de bouleversement du bien-être social des populations rurales (Hadonou-Yovo et al., 2019). Ainsi, documenter le savoir traditionnel concernant les plantes à usage artisanal en vue de leur préservation serait une solution au problème. Au Centre-est et au Nord-est de la Côte d'Ivoire à cheval entre la forêt semi-caducifoliée et le secteur préforestier du domaine guinéen se trouvent quatre tribus Agni, formant une limite linguistique intermédiaire entre les peuples Djimini, Koulango au Nord et les grands Royaumes Djuablin et Indenié, plus au Sud. A notre connaissance, les savoirs et savoir-faire en matière d'utilisation des plantes de ces peuples ont été très peu documentés. Tout usage d'une plante est lié au comportement socioculturel des communautés (Yetein et al., 2013). Cette étude vise à valoriser le savoir traditionnel sur les plantes à usage artisanal chez ces sous-groupes Agni. De façon spécifique, il s'agit de recenser et évaluer l'importance interculturelle des plantes à usage artisanal au sein de ces tribus ; déterminer le niveau de partage des connaissances sur les plantes à usage artisanal entre ces quatre tribus.

\section{Materiel Et Methodes}

\section{Présentation du milieu d'étude}

L'étude a été conduite dans trois Départements (Daoukro, Koun-fao et Sandegué) du Centre-est et Nord-est de la Côte d'Ivoire (Figure 1) de mars 2016 à septembre 2019. Elle a concerné quatre sous-préfectures et 12 villages, que sont : les villages d'Ettrokro, Katimansou et Zanzansou pour les AgniAbè (Sous-préfecture d'Ettrokro), les villages de Sanguehi, Kamélé et Kouakoukankro pour les Agni-Barabo (Sous-préfecture de Sandégué), les villages de Senandé, Yakassé-Bini et Missoumihian 1 pour les Agni-Bini (Sous-préfecture de Kouassi-Datékro,) et les villages de Kokomian, 
Bossignanienkro et Sogoyaokro pour les Agni-Bona (Sous-préfecture de Kokomian).

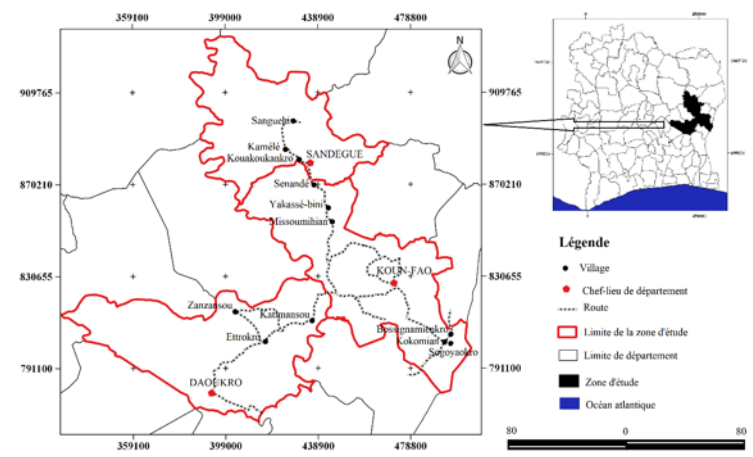

Figure 1.Carte de la zone d'étude (Département de Daoukro ; Koun-fao et Sandegué)

\section{Matériel d'étude}

Le matériel d'étude pour cette investigation est constitué de matériels standard d'enquête ethnobotanique (une fiche d'enquête, un appareil photo, le matériel classique de récolte et de conservation d'échantillons) et l'ensemble des plantes utilisées par les différentes populations locales dans l'artisanat.

\section{Méthode de collecte des données}

L'enquête ethnobotanique a été réalisée en deux étapes à l'aide d'un guide d'entretien. La première étape a consisté en un entretien semi-structuré à l'aide d'un questionnaire au cours d'un "porte-à-porte » aléatoire dans des foyers de chaque village. Les entretiens se sont focalisés sur les plantes spontanées utilisées pour construire des habitats traditionnels et pour fabriquer différents outils ou articles utilisés au quotidien par les informateurs. Les informations recherchées sont : le nom local des plantes, les parties utilisées et l'objet fabriqué. Cette première partie a concerné 571 informateurs répartis comme suit : 174 Agni-Abè (71 femmes et 103 hommes), 182 Agni-Barabo (92 femmes et 90 hommes), 118 Agni-Bini (68 femmes et 50 hommes) et 96 Agni-Bona (61 femmes et 35 Hommes). Pour la seconde étape, des randonnées dans les «brousses » environnantes avec des informateurs ont été effectuées. Cette étape a permis de compléter la liste des plantes recueillie lors de la première étape et de constituer un herbier. L'identification des plantes a été faite au Laboratoire de Botanique à l'Université Nangui Abrogoua. Les noms scientifiques ont été mis à jour à l'aide de la base de données African Plants Database (version 3.4.0), consultée le 20/10/2020.

\section{Catégorisation des usages des plantes artisanales}

Selon l'objet fabriqué et son utilité (artistique, mobilière ou ludique), l'usage des plantes a été subdivisé en quatre catégories : 
- La construction des habitats traditionnels (pilier, charpente, toiture).

- La confection des meubles (chaises, table, lits, etc.).

- La confection des ustensiles de cuisine (écuelles, paniers, mortiers, pilons, spatules).

- La fabrication des objets d'art et de jeux (sculptures, instruments de musique, etc.).

\section{Analyse des données}

Différents indices et traitements statistiques ont servi à évaluer l'importance culturelle et le partage des connaissances sur l'usage artisanal des plantes. Ainsi :

Le diagramme de Venn a été utilisé pour montrer la distribution des plantes à usage artisanal entre les différentes tribus.

L'indice d'importance culturelle ou Cultural importance index (IC) a été utilisé pour évaluer le niveau d'importance de chaque espèce dans une culture donnée (Tardio \& Pardo-de-Santayana, 2008). Il est calculé selon la formule suivante :

$$
I C=\sum_{U=U_{1}}^{U_{N C}} \sum_{I=1}^{I_{N}} \frac{U R_{U I}}{N}
$$

Où, $U R$ : Nombre de personnes ayant mentionné un usage donné, $N C$ : Nombre total de catégories d'usages, $N$ : Nombre total d'informateurs. Une valeur élevée d'IC indique une importance culturelle élevée pour la plante donnée.

Le test de $\mathrm{Khi}^{2}$ a été utilisé pour comparer l'homogénéité des richesses spécifiques aux fins d'évaluer la significativité des différences entres les tribus. Le seuil de significativité est $\alpha=0,05$.

Les valeurs de l'IC ont servi à faire l'analyse factorielle des correspondants pour évaluer le niveau du partage de connaissances entre les différents peuples Agni visités.

Puis les données de présence absence ont été utilisées pour évaluer la concordance des usages artisanaux, entre les quatre communautés, le coefficient de similarité de Jaccard (Jaccard, 1908) a été utilisé pour comparer les connaissances sur les plantes utilisées, dans l'artisanat par ces différentes tribus. Ce coefficient a été calculé selon la formule suivante :

$$
S_{i j}=\frac{a}{a+b+c}
$$

Où $S_{i j}$ est le coefficient de Jaccard, $a$ le nombre d'espèces communes aux groupes $i$ et $j ; b$ le nombre d'espèces présentes uniquement dans le groupe $i, c$ le nombre d'espèces uniquement dans le groupe $j$. Les analyses de données ont été réalisées avec le logiciel R (version 3.6.1). 


\section{Resultats}

\section{Diversité et distribution des espèces végétales à usage artisanal}

Pour l'ensemble des quatre tribus Agni, 104 espèces utilisées dans l'artisanat ont été recensées. Elles se répartissent en 95 genres et 35 familles dont les plus importantes numériquement, sont les Leguminosae (17 espèces), les Malvaceae (13 espèces) et les Apocynaceae (sept espèces). Cette richesse spécifique varie d'une tribu à une autre, avec cependant 14 plantes (soit 13,46\%) communes aux quatre tribus (Figure 2). Il s'agit de Afzelia africana Sm. ex Pers. (Leguminosae), Alstonia boonei De Wild. (Apocynaceae), Antiaris africana Engl. (Moraceae), Ceiba pentandra (L.) Gaertn. (Malvaceae), Elaeis guineensis Jacq. (Arecaceae), Funtumia elastica (P. Preuss) Stapf (Apocynaceae), Holarrhena floribunda (G. Don) T. Durand \& Schinz (Apocynaceae), Mansonia altissima (A.Chev.) A. Chev. (Malvaceae), Margaritaria discoidea (Baill.) G. L. Webster (Phyllanthaceae), Milicia exceslsa (Welw.) C. C. Berg, (Moraceae), Morus mesozygia Stapf (Moraceae), Picralima nitida (Stapf) T. Durand \& H. Durand (Apocynaceae), Ricinodendron heudelotii (Baill.) Pierre ex Heckel (Euphorbiaceae), Trema orientalis (L.) Blume (Cannabaceae). Les plantes sont recherchées essentiellement pour trois parties : le tronc (93\%), les feuilles (5\%) et les fruits $(2 \%)$.

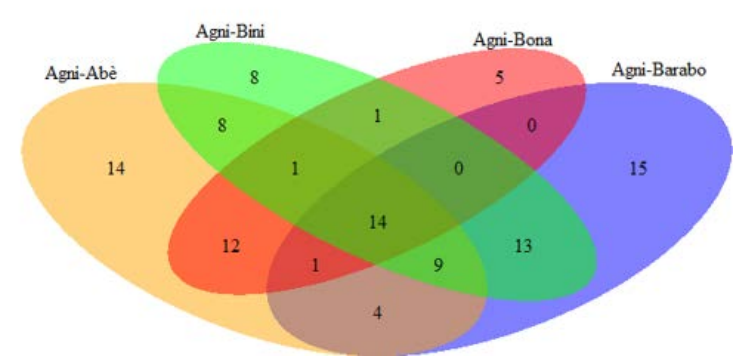

Figure 2. Diagramme de Venn représentant la distribution des plantes à usage artisanal entre les tribus Agni étudiées

\section{Usage artisanal des plantes}

Parmi les 104 espèces à usage artisanal recensées, 74 espèces sont utilisées pour bâtir des huttes, 39 espèces pour la confection de meubles traditionnels, 73 espèces pour la confection des articles de ménage et 37 pour la confection des objets arts et culturels. Ces nombres varient selon les tribus (Figure 3). Selon le type d'usage (construction, meuble traditionnel, articles de ménage, objets arts et culturels) et le rôle du matériel végétal, les plantes utilisées sont différentes d'une tribu à l'autre. Ainsi, Anogeissus leiocarpa (DC.) Guill. \& Perr. (Combretaceae), Crossopteryx febrifuga (Afzel. ex G.Don) Benth. (Rubiaceae), Terminalia schimperiana Hochst. (Combretaceae) avec les feuilles de Andropognon gayanus Kunth (Poaceae) 
chez les Agni-Barabo et les Agni-Bini (Figure 4A) puis, Terminalia superba Engl. \& Diels (Combretaceae), Mansonia altissima (A.Chev.) A. Chev. (Malvaceae), avec les feuilles tissées de Raphia hookeri G.Mann \& H. Wendl (Arecaceae) chez les Agni-Abè et les Agni-Bona sont utilisées pour bâtir des habitats traditionnels.

Les plantes les plus sollicitées dans la sous-catégorie meuble traditionnel sont : Holarrhena floribunda (G.Don) T. Durand \& Schinz (Apocynaceae), Ricinodendron heudelotii (Baill.) Pierre ex Heckel (Euphorbiaceae), Baissea multiflora, Elaeis guineensis et Raphia hookeri.

Les plantes les plus sollicitées dans la sous-catégorie des articles de ménage sont: Afzelia africana Sim.ex Pers. (Leguminosae), Nauclea diderrichii (De Wild. \& T. Durand) Merr. (Rubiaceae), Khaya senegalensis (Desr.) A. Juss. (Meliaceae), Nesogordonia papaverifera (A.Chev.) Capuron ex N. Hallé (Malvaceae), Vitellaria paradoxa C. F. Gaertn. (Sapotaceae), Pseudocedrela kotschyi (Schweinf.) Harms, (Meliaceae), Zanthoxylum zanthoxyloides (Lam.) Zepern. \& Timler (Rutaceae), etc. enfin le panier avec Baissea multiflora et Elaeis guineensis (Figure 4 B).

Les plantes les plus sollicitées dans la sous-catégorie d'objets d'art et culturelles sont : Anthocleista nobilis G.Don (Meliaceae), Cussonia arborea Hockst. ex A. Rich (Araliaceae), Cordia millenii Baker (Boraginaceae) sont utilisées pour la confection des Tam-tam. Holarrhena floribunda, Ricinodendron heudelotii, Alstonia boonei pour les manches de fusil et les statuettes.

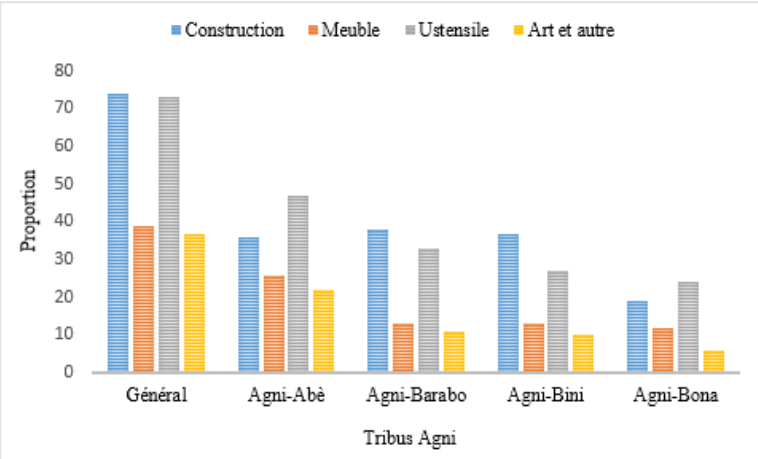

Figure 3. Catégories d'usages des plantes à usage artisanal en fonction des tribus 

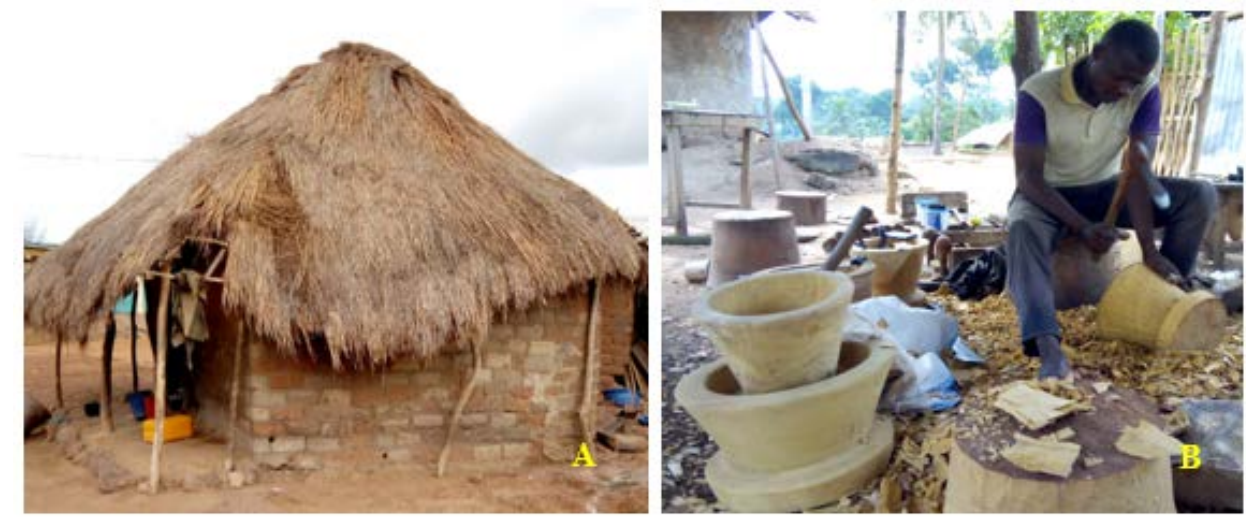

Figure 4. Quelques plantes à usage artisanal : A. Toiture de hutte confectionnée avec Andropogon gayanus à Senandé (Agni-Bini) ; B. Artisan confectionnant un mortier avec Nauclea diderrichii à Kokomian (Agni-Bona)

\section{Importance culturelle et variation interculturelle}

De cette investigation il ressort que, 15 espèces $(14,42 \%)$ ont un indice d'importance culturelle élevé (IC $\geq 0,5)$. Les valeurs et les plantes importantes culturellement varient selon les communautés (Tableau 1). Par exemple, chez les Agni-Abè Afzelia africana (IC $=0.5$ ), Baissea multiflora (IC $=0.5$ ), Mansonia altissima (IC = 0,56), Picralima nitida ( $\mathrm{IC}=0,58)$, Milicia exceslsa (IC $=0,59)$, Holarrhena floribunda (IC $=0,76$ ), Cordia millenii (IC $=0.77$ ) et Nesogordonia papaverifera (IC $=1,04$ ) sont plus important, tandis que chez les Agni-Barabo c'est Holarrhena floribunda (IC = 0,57), Zanthoxylum zanthoxyloides (IC $=0,5)$, Khaya senegalensis $(\mathrm{IC}=0,69)$, Pseudocedrela kotschyi (IC $=0,67$ ) et Vitellaria paradoxa (IC $=1,05)$ qui sont plus important. Chez les Agni-Bini c'est Holarrhena floribunda (IC = 1,52), Pseudocedrela kotschyi (IC $=0,71)$, Vepris heterophylla $(\mathrm{IC}=0,58)$ et Zanthoxylum zanthoxyloides (IC $=0,51$ ) sont les plus importants. Tandis que les plus importantes chez les Agni-Bona sont Celtis zenkeri $(\mathrm{IC}=0,51)$ et Nauclea diderrichii ( $\mathrm{IC}=0,71)$. Ces plantes sont utilisées dans plusieurs types d'usages.

Tableau 1. Importance culturelle des plantes à usage artisanal

\begin{tabular}{|l|l|c|c|c|c|}
\hline & & \multicolumn{4}{|c|}{ IC } \\
\hline Espèces végétales & Familles & $\begin{array}{c}\text { Agni- } \\
\text { Abè }\end{array}$ & $\begin{array}{c}\text { Agni- } \\
\text { Barabo }\end{array}$ & $\begin{array}{c}\text { Agni- } \\
\text { Bini }\end{array}$ & $\begin{array}{c}\text { Agni- } \\
\text { Bona }\end{array}$ \\
\hline Afzelia africana Sm. ex Pers. & Leguminosae & 0,50 & 0,07 & 0,25 & 0,16 \\
\hline $\begin{array}{l}\text { Alchornea cordifolia (Schumach. \& } \\
\text { Thonn.) Müll. Arg. }\end{array}$ & Euphorbiaceae & - & - & - & 0,15 \\
\hline Alstonia boonei De Wild. & Apocynaceae & 0,35 & 0,07 & 0,31 & 0,19 \\
\hline Andropognon gayanus Kunth & Poaceae & - & 0,75 & 0,88 & - \\
\hline Anogeissus leiocarpa (DC.) Guill. \& Perr. & Combretaceae & - & 0,12 & 0,22 & - \\
\hline Anthocleista nobilis G.Don & Meliaceae & - & 0,02 & 0,10 & - \\
\hline Antiaris africana Engl. & Moraceae & 0,12 & 0,01 & 0,02 & 0,08 \\
\hline
\end{tabular}




\begin{tabular}{|c|c|c|c|c|c|}
\hline Azadirachta indica A. Juss. & Meliaceae & 0,17 & 0,06 & - & - \\
\hline Baissea multiflora A. DC. & Apocynaceae & 0,50 & - & 0,04 & 0,26 \\
\hline Bambusa vulgaris Schrad. ex J. C. Wendl. & Poaceae & 0,13 & 0,12 & 0,02 & - \\
\hline Baphia nitida Lodd. & Apocynaceae & - & - & - & 0,35 \\
\hline Baphia pubescens Hook. f. & Leguminosae & - & - & 0,05 & - \\
\hline Blighia sapida K. D. Koenig & Leguminosae & - & 0,09 & 0,02 & - \\
\hline Blighia welwitschii (Hiern) Radlk. & Leguminosae & - & 0,01 & - & - \\
\hline Bombax buonopoizense P. Beauv. & Malvaceae & 0,06 & 0,02 & 0,08 & - \\
\hline Borassus aethiopum Mart. & Arecaceae & 0,03 & - & 0,06 & - \\
\hline Bridelia ferruginea Benth. & Phyllanthaceae & - & - & 0,21 & - \\
\hline Campylostemon laurentii De Wild. & Celastraceae & 0,43 & 0,02 & - & - \\
\hline Cassia sieberiana DC. & Leguminosae & - & 0,03 & - & - \\
\hline Ceiba pentandra (L.) Gaertn. & Malvaceae & 0,32 & 0,14 & 0,16 & 0,06 \\
\hline Celtis zenkeri Engl. & Cannabaceae & 0,01 & - & 0,02 & 0,51 \\
\hline $\begin{array}{l}\text { Chionanthus africanus (Welw.ex Knobl.) } \\
\text { Steam }\end{array}$ & Olacaceae & 0,04 & - & - & - \\
\hline Cnestis ferruginea DC. & Connaraceae & - & 0,14 & 0,19 & - \\
\hline $\begin{array}{l}\text { Cola gigantea var. glabrescens Brenan \& } \\
\text { Keay }\end{array}$ & Malvaceae & 0,02 & - & - & 0,05 \\
\hline Cola millenii K. Schum. & Malvaceae & 0,16 & - & - & - \\
\hline $\begin{array}{l}\text { Combretum nigricans Lepr. ex Guill. \& } \\
\text { Perr. }\end{array}$ & Combretaceae & 0,34 & - & 0,07 & - \\
\hline Cordia millenii Baker & Boraginaceae & 0,85 & - & 0,41 & 0,29 \\
\hline Crescentia cujete L. & Bignoniaceae & 0,07 & - & - & - \\
\hline $\begin{array}{l}\text { Crossopteryx febrifuga (Afzel. ex G.Don) } \\
\text { Benth. }\end{array}$ & Rubiaceae & - & 0,09 & - & - \\
\hline Cussonia arborea Hockst. ex A.Rich & Araliaceae & - & 0,17 & - & - \\
\hline Cuviera acutiflora DC. & Rubiaceae & 0,01 & - & - & - \\
\hline Cynometra megalophylla Harms & Leguminosae & 0,06 & - & 0,03 & - \\
\hline Daniellia oliveri (Rolfe) Hutch. \& Dalziel & Rubiaceae & - & 0,04 & - & - \\
\hline Detarium microcarpum Guill. \& Perr. & Leguminosae & - & 0,02 & 0,26 & - \\
\hline Detarium senegalense J. F. Gmel. & Leguminosae & - & 0,01 & - & - \\
\hline Dialium guineense Willd. & Leguminosae & 0,37 & 0,27 & 0,14 & - \\
\hline $\begin{array}{l}\text { Diospyros mespiliformis Hochst. ex } \\
\text { A.DC. }\end{array}$ & Ebenaceae & - & 0,14 & 0,35 & - \\
\hline Elaeis guineensis Jacq. & Arecaceae & 0,35 & 0,01 & 0,05 & 0,38 \\
\hline $\begin{array}{l}\text { Entandrophragma utile (Dawe \& } \\
\text { Sprague) Sprague }\end{array}$ & Meliaceae & - & - & - & 0,05 \\
\hline Eremospatha dransfieldii Sunderl. & Arecaceae & 0,15 & - & - & 0,08 \\
\hline $\begin{array}{l}\text { Erythrophleum suaveolens (Guill. \& } \\
\text { Perr.) Brenan }\end{array}$ & Leguminosae & 0,30 & 0,14 & - & - \\
\hline Ficus sur Forssk. & Moraceae & - & - & 0,24 & - \\
\hline Funtumia elastica (P. Preuss) Stapf & Apocynaceae & 0,06 & 0,15 & 0,08 & 0,25 \\
\hline Garcinia afzelii Engl. & Clusiaceae & 0,12 & 0,01 & 0,01 & - \\
\hline Grewia megalocarpa Juss. & Malvaceae & - & 0,02 & - & - \\
\hline $\begin{array}{l}\text { Griffonia simplicifolia (Vahl ex DC.) } \\
\text { Baill. }\end{array}$ & Leguminosae & 0,02 & - & - & - \\
\hline Hildegardia barteri (Mast.) Kosterm. & Malvaceae & 0,01 & - & - & - \\
\hline
\end{tabular}




\begin{tabular}{|c|c|c|c|c|c|}
\hline $\begin{array}{l}\text { Holarrhena floribunda (G.Don) T. } \\
\text { Durand \& Schinz }\end{array}$ & Apocynaceae & 0,77 & 0,57 & 1,51 & 0,96 \\
\hline Hymenocardia acida Tul. & Phyllanthaceae & - & - & 0,02 & - \\
\hline Hypselodelphys triangularis Jongkind & Maranthaceae & 0,17 & - & - & - \\
\hline Khaya ivorensis A.Chev. & Meliaceae & 0,24 & - & - & 0,06 \\
\hline Khaya senegalensis (Desr.) A. Juss. & Meliaceae & - & 0,69 & 0,22 & - \\
\hline Lagenaria siceraria (Molina) Standl. & Curcubitaceae & 0,06 & 0,01 & 0,03 & - \\
\hline Lasiodiscus chevalieri Hutch. & Rhamnaceae & 0,05 & - & - & - \\
\hline Lasiodiscus mannii Hook. f. & Rhamnaceae & - & - & 0,06 & - \\
\hline Lecaniodiscus cupanioides Planch. & Sapindaceae & 0,02 & 0,03 & 0,07 & - \\
\hline Leptonychia pubescens Keay & Malvaceae & - & - & 0,37 & 0,03 \\
\hline Mangifera indica L. & Anacardiaceae & 0,01 & - & 0,01 & - \\
\hline Mansonia altissima (A.Chev.) A.Chev. & Malvaceae & 0,59 & 0,19 & 0,01 & 0,11 \\
\hline $\begin{array}{l}\text { Marantochloa congensis (K. Schum.) J. } \\
\text { Léonard \& Mullend. }\end{array}$ & Marantaceae & 0,28 & - & - & - \\
\hline $\begin{array}{l}\text { Margaritaria discoidea (Baill.) G.L. } \\
\text { Webster }\end{array}$ & Phyllanthaceae & 0,04 & 0,04 & 0,03 & 0,13 \\
\hline Milicia excelsa (Welw.) C. C. Berg & Moraceae & 0,59 & 0,18 & 0,52 & 0,51 \\
\hline Morus mesozygia Stapf & Moraceae & 0,19 & 0,01 & 0,01 & 0,31 \\
\hline Musanga cecropioides R. Br. & Urticaceae & 0,30 & - & 0,02 & - \\
\hline $\begin{array}{l}\text { Nauclea diderrichii (De Wild. \& T. } \\
\text { Durand) Merr. }\end{array}$ & Rubiaceae & 0,13 & - & - & 0,71 \\
\hline $\begin{array}{l}\text { Nesogordonia papaverifera (A.Chev.) } \\
\text { Capuron ex N. Hallé }\end{array}$ & Malvaceae & 1,05 & 0,16 & - & 0,36 \\
\hline $\begin{array}{l}\text { Newbouldia laevis (P. Beauv.) Seem. ex } \\
\text { Bureau }\end{array}$ & Bignoniaceae & 0,06 & - & - & - \\
\hline Olax subscorpioidea Oliv. & Olacaeae & - & 0,02 & 0,03 & - \\
\hline Parkia biglobosa (Jacq.) R. Br. ex G.Don & Leguminosae & 0,01 & - & - & - \\
\hline $\begin{array}{l}\text { Picralima nitida (Stapf) T. Durand \& H. } \\
\text { Durand }\end{array}$ & Apocynaceae & 0,58 & 0,10 & 0,44 & 0,16 \\
\hline Piliostigma reticulatum (DC.) Hochst. & Leguminosae & - & 0,05 & - & - \\
\hline Pouteria alnifolia (Baker) Roberty & Sapotaceae & - & 0,05 & - & - \\
\hline $\begin{array}{l}\text { Pseudocedrela kotschyi (Schweinf.) } \\
\text { Harms }\end{array}$ & Meliaceae & - & 0,67 & 0,78 & - \\
\hline Psydrax subcordata (DC.) Bridson & Rubiaceae & - & 0,12 & - & - \\
\hline Pterocarpus erinaceus Poir. & Leguminosae & - & 0,02 & - & - \\
\hline Pterygota macrocarpa K. Schum. & Malvaceae & 0,35 & - & - & - \\
\hline Pycnanthus angolensis (Welw.) Warb. & Myristicaceae & 0,27 & - & - & 0,06 \\
\hline Raphia hookeri G.Mann \& H. Wendl. & Arecaeae & 0,01 & 0,01 & - & - \\
\hline Rauvolfia vomitoria Afzel. & Apocynaceae & 0,01 & - & - & 0,05 \\
\hline $\begin{array}{l}\text { Ricinodendron heudelotii (Baill.) Pierre } \\
\text { ex Heckel }\end{array}$ & Euphorbiaceae & 0,40 & 0,28 & 0,14 & 0,30 \\
\hline Samanea dinklagei (Harms) Keay & Leguminosae & 0,04 & - & - & - \\
\hline $\begin{array}{l}\text { Scytopetalum tieghemii (A.Chev.) Hutch. } \\
\text { \& Dalziel }\end{array}$ & Lecythidaceae & - & - & 0,13 & - \\
\hline $\begin{array}{l}\text { Sericanthe toupetou (Aubrév. \& Pellegr.) } \\
\text { Robbr. }\end{array}$ & Rubiaceae & - & - & 0,01 & 0,13 \\
\hline Spathodea campanulata P. Beauv. & Bignoniaceae & - & 0,06 & - & - \\
\hline Spondias mombin L. & Anacardiaceae & 0,04 & 0,05 & 0,04 & - \\
\hline
\end{tabular}




\begin{tabular}{|l|l|c|c|c|c|}
\hline Sterculia rhinopetala K. Schum. & Malvaceae & 0,02 & - & - & 0,13 \\
\hline Sterculia tragacantha Lindl. & Malvaceae & 0,02 & - & - & - \\
\hline Talbotiella gentii Hutch. \& Greenway & Leguminosae & 0,22 & - & - & - \\
\hline Tapura ivorensis Breteler & Dichapetalaceae & 0,08 & - & - & - \\
\hline Tectona grandis L. F. & Lamiacaeae & - & - & 0,02 & - \\
\hline Terminalia ivorensis A.Chev. & Combretaceae & 0,23 & - & - & 0,05 \\
\hline Terminalia schimperiana Hochst. & Combretaceae & - & 0,31 & 0,19 & - \\
\hline Terminalia superba Engl. \& Diels & Combretaceae & 0,08 & - & - & 0,23 \\
\hline $\begin{array}{l}\text { Tetrapleura tetraptera (Schumach. \& } \\
\text { Thonn.) Taub. }\end{array}$ & Leguminosae & - & - & - & 0,09 \\
\hline Thaumatococcus daniellii (Benn.) Benth. & Marantaceae & 0,06 & - & - & - \\
\hline Treculia africana Decne. & Moraceae & - & 0,02 & - & - \\
\hline Trema orientalis (L.) Blume & Cannabaceae & 0,16 & 0,02 & 0,06 & 0,11 \\
\hline Triplochiton scleroxylon K. Schum. & Malvaceae & 0,30 & - & 0,04 & 0,01 \\
\hline Vepris heterophylla (Engl.) Letouzey & Rutaceae & 0,02 & 0,48 & 0,63 & - \\
\hline Vitellaria paradoxa C. F. Gaertn. & Sapotaceae & 0,01 & 1,05 & 0,46 & - \\
\hline Vitex doniana Sweet & Lamiaceae & - & 0,01 & - & - \\
\hline Xylopia quintasii Engl. \& Diels & Annonaceae & - & 0,16 & 0,06 & - \\
\hline Zanthoxylum leprieurii Guill. \& Perr. & Rutaceae & - & 0,01 & - & - \\
\hline $\begin{array}{l}\text { Zanthoxylum zanthoxyloides (Lam.) } \\
\text { Zepern. \& Timler }\end{array}$ & Rutaceae & - & 0,52 & 0,64 & - \\
\hline
\end{tabular}

\section{Concordance des usages artisanaux entre les quatre tribus}

Le nombre de plantes citées dans l'artisanat par les quatre tribus est 63 plantes (les Agni-Abè), 56 plantes (les Agni-Barabo), 54 plantes (les AgniBini) et 34 plantes (les Agni-Bona) a été comparé à l'aide d'un test de Khi². Cette analyse révèle des similitudes et des différences significatives entre certaines tribus comme l'illustre le Tableau 2. La différence la plus forte est obtenue entre les Agni-Abè et les Agni-Bona ( $p=0,002)$, ainsi qu'entre AgniBarabo et Agni-Bona ( $p=0,0105)$. Par contre, le nombre de plantes citées par les Agni-Abè, les Agni-Barabo et les Agni-Bini est quasi identique $(p>0,05)$.

Tableau 2. Comparaison des richesses spécifiques par communautés

\begin{tabular}{|l|l|l|l|}
\hline & p-value & \multicolumn{2}{l|}{} \\
\hline & Agni-Abè & Agni-Barabo & Agni-Bini \\
\hline Agni-Abè & - & - & - \\
\hline Agni-Barabo & 0,521 & - & - \\
\hline Agni-Bini & 0,357 & 0,849 & - \\
\hline Agni-Bona & 0,002 & 0,0105 & 0,033 \\
\hline
\end{tabular}

Concernant les usages, l'analyse du coefficient de Jaccard (Tableau 3) montre également de fortes différences entre les quatre tribus de façon générale $(S \leq 0,5)$ et de façon plus prononcée entre Agni-Barabo et Agni-Bona $(S=0,195)$.

Tableau 3. Niveau de partage des connaissances

\begin{tabular}{|c|c|c|c|}
\hline & & S & \\
\hline & Agni-Abè & Agni-Barabo & Agni-Bini \\
\hline
\end{tabular}




\begin{tabular}{|c|c|c|c|}
\hline Agni-Barabo & 0,308 & & \\
\hline Agni-Bini & 0,365 & 0,48 & \\
\hline Agni-Bona & 0,386 & 0,195 & 0,29 \\
\hline
\end{tabular}

Ces différences sont aussi relevées par l'analyse factorielle des correspondants (Figure 5). Elle a permis de repartir les espèces en quatre groupes suivant l'indice d'importance culturelle. Le premier groupe (G1) est constitué des plantes citées par les Agni-Abè. Le deuxième groupe (G2) est composé de plantes citées les Agni-Barabo. Le troisième groupe (G3) est formé par les plantes citées par les Agni-Bini et le quatrième groupe (G4) est formé par les plantes citées par les Agni-Bona. Par ailleurs, ce résultat est très significatif $\left(\mathrm{Khi}^{2}=3056,791 ; \mathrm{p}\right.$-value $\left.=0\right)$.

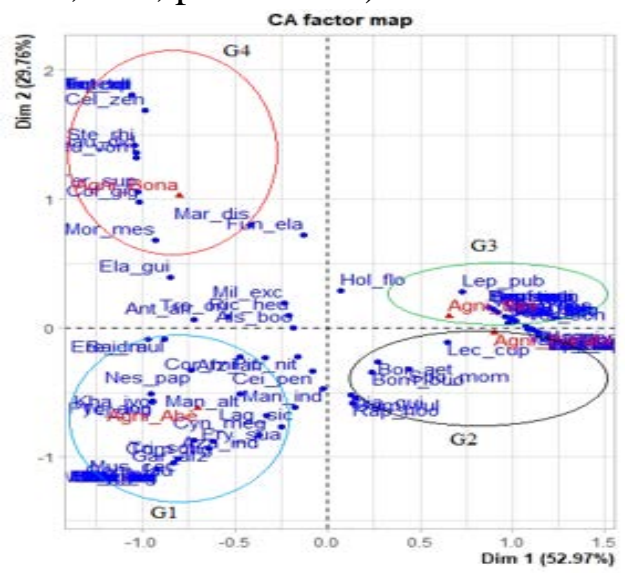

Figure 5. comparaison des savoirs des quatre tribus Agni

\section{Discussion}

Cette étude ethnobotanique sur la valorisation du savoir traditionnel au niveau des plantes à usage artisanal chez quatre tribus Agni a permis de répertorier 104 plantes. Ces plantes sont diverses et leurs usages reflètent le paysage végétal local. Les modèles d'habitations traditionnels et les différents objets confectionnés sont la traduction concrète du savoir et savoir-faire de ces populations dans la maîtrise des ressources de leur milieu naturel (Kouakou, 2019 ; Kouakou et al., 2020). Les espèces utilisées varient selon les tribus, avec les Agni-Abè en tête et les Agni-Bona en dernière position. Cette différence de diversité floristique pourrait s'expliquer par deux raisons majeures. D’abord, les quatre tribus appartiennent à deux types de végétations différentes. Ce sont : les forêts semi-caducifoliées et le secteur préforestier qui se caractérisent par la transition forêts-savanes avec une nette dominance des formations de savanes. La dégradation de l'environnement due à un défrichement intensif, et cela est plus prononcée chez les Agni-Bona. En effet, les coupes répétées des espèces utiles ajoutées à l'intensification des activités 
anthropiques (l'exploitation forestière anarchique, agriculture itinérante sur abattis-brûlis, etc.) à l'absence d'autorité de surveillances a entrainé la raréfaction des ressources utiles et avec elle la perte de savoir et savoir-faire. La variation de la diversité au niveau de la richesse spécifique a été aussi observée en Côte d'Ivoire et ailleurs en Afrique de l'Ouest. En effet, les peuples Koulango et Lobi ont mentionné un nombre de plantes à usage artisanal différent malgré le partage de la même végétation savanicole (Kouakou, 2019). En outre, au Sénégal la diversité est plus importante au Sud (Kahi) comparé au Nord (Mérima Dakhar) du bassin arachidier et cela à cause de la différence de végétation, mais aussi de la dégradation de l'environnement (Ndiaye et al., 2017).

Comme en zone sahélienne, les tribus Agni ont adopté des types d'habitats adaptés à leur mode de vie. Ces modèles d'habitation sont la traduction concrète du savoir-faire des populations locales dans la maîtrise des ressources de leur milieu naturel (Kouakou et al., 2020). La sélection du matériel végétal utilisée se fait en fonction de certains critères (Kimpouni \& Nguembo, 2018). Les plantes sont sélectionnées en fonction de la perception de la solidité et la durabilité du bois, sa résistance à la pourriture aux termites et aux insectes xylophages, la qualité de droiture et de facilité à travailler du bois, l'abondance de la plante dans le milieu (Kimpouni \& Nguembo, 2018). La perception de ces différents caractères est fonction des tribus comme l'a mentionné Kouakou (2019). Par conséquent, les plantes utilisées ou leurs usages varient en fonction de la communauté. Des observations semblables ont été faites par Soro et al. (2014) en Côte d'Ivoire et Lougbegnon et al. (2011) au Bénin. Ces auteurs révèlent que les peuples ruraux du Sud-ouest de la Côte d'Ivoire ainsi que, ceux de la vallée de Sitatunga au Bénin utilisent de ressources végétales à leur disposition pour construire des habitats traditionnels (cuisine, hutte, etc.).

De ces investigations, il ressort que les quatre tribus Agni visitées ont une faible diversité en mobilier traditionnel. Toutefois, ces tribus font une sélection de plantes en fonction de l'objet confectionné. Les plantes les plus recherchées sont Holarrhena floribunda, Ricinodendron heudelotii et Alstonia boonei pour la confection de mobiliers. Ces mobiliers sont constitués pour l'essentiel de tabouret et de table. Les chaises et les hamacs sont fabriqués avec Baissea multiflora, Elaeis guineensis et Raphia hookeri. Des observations semblables ont été faites par Malan (2008) chez les Ehotilé un peuple culturellement proche des Agni de notre zone d'étude.

Au niveau de la fabrication des objets et ustensiles, les critères comme la maniabilité, la résistance aux intempéries ainsi que la qualité physique sont recherchés chez les végétaux utilisés (Kouakou, 2019). En effet, les principaux ustensiles de cuisine sont : le panier, l'assiette, le pilon et le mortier. Ces ustensiles sont le reflet du régime alimentaire de ces tribus Agni ainsi que du 
peuple Akan en général. En fonction du type d'ustensile fabriqué, l'artisan est soumis à un choix. Ainsi, Baissea multiflora, Elaeis guineensis et Raphia hookeri sont utilisées pour confectionner diverses sortes de paniers, avec pour rôle le transport et le stockage des vivres (Zeh, 2018). Par ailleurs, Celtis zenkeri, Picralima nitida, et Nesogordonia papaverifera sont les plus utilisées pour le pilon. Alors que, Khaya senegalensis, Milicia exceslsa, Nauclea diderrichii et Vitellaria paradoxa sont les plus recherchés pour la fabrication des mortiers. Ce résultat s'expliquerait par le fait que ces arbres sont solides et donnent des articles ayant une longue durée comme souligné par Vroh bi et al. (2014), Ouattara et al. (2016) et Kouakou (2019).

Les instruments de musique, les articles d'arts et utilitaire chez les tribus Agni du Nord-est au Centre-est ne diffèrent pas des autres groupes Akan (Malan, 2008). Par contre, les plantes utilisées pour confectionner ces articles varient d'une tribu à autre. Le tam-tam, principal instrument de musique Akan est taillé dans le bois de Cussonia arborea et Anthocleista nobilis chez les Agni-Barabo et Agni-Bini. Cependant, chez les Agni-Abè et les Agni-Bona cet instrument ludique est taillé dans le bois de Cordia millenii. De plus, Holarrhena floribunda, Ricinodendron heudelotii, Alstonia boonei sont utilisées par toutes ces tribus pour confectionner les manches de fusil traditionnel, ainsi que des statuettes. Les rites et rituels sont toujours d'actualités avec les statuettes, les tam-tams parleurs et autres.

Les plantes à usage artisanal ont dans l'ensemble une importance culturelle pour les différentes communautés. Cependant, certaines plantes sont plus importantes que d'autres au sein de chaque communauté. Ces plantes avec IC $\geq 0,5$ représentent $14,42 \%$ du total des plantes citées. Ce faible nombre de plantes ayant un indice d'importance culturelle élevé peut se justifier en premier lieu, par le fait que ces plantes doivent respecter un ensemble de caractéristiques recherchées par les artisans. En deuxième lieu, par la nondisponibilité des ressources naturelles utiles pour l'artisanat. Enfin, par la concurrence des outils et matériaux modernes disponibles de plus en plus sur les marchés locaux. Ces observations ont été faites par Kouakou et al. (2020) qui révèlent que seulement $9 \%$ des plantes citées par les Koulango et Lobi ont une fréquence de citation supérieure à $50 \%$. En général, les espèces de plantes culturellement importantes pour ces communautés sont celles à usage multiple qui interviennent dans presque tous les types d'usage (Gning et al., 2013). Seule Holarrhena floribunda avec un IC élevé, est utilisée intensivement par ces différentes tribus Agni dans tous les types usages artisanaux. Ayant la tendresse et la légèreté des bois d'Apocynaceae, Holarrhena floribunda est taillée pour des usages multiples dans la vie quotidienne (Malan, 2008).

La détermination du niveau de concordance des connaissances sur les plantes à usage artisanal entre tribus a montré de fortes différences entre les quatre tribus de façon générale. Ces différences peuvent s'expliquer par le 
faite que la végétation est différente du Nord au Sud de la zone d'étude. Ensuite, les réalités socioculturelles et la promiscuité entre les tribus peuvent avoir un incident sur la connaissance et l'usage des plantes (Hodonou-Yovo et al., 2019 ; Malan et al., 2020). De plus, la disponibilité et l'accessibilité des ressources végétales influent sur leur exploitation (Kouakou et al., 2017). Des observations semblables ont été faites en Côte d'Ivoire par Kouakou et al. (2020) et au Bénin par Hodonou-Yovo et al. (2019). En effet, au Nord-est de la Côte d'Ivoire des différences ont été observées au niveau de l'usage artisanal des plantes entre les peuples Koulango et Lobi, et selon HodonouYovo et al. (2019) les différences communautés de la périphérie de la Réserve de biosphère du Mono au Bénin s'accordent moyennement sur les plantes utilisées dans l'artisanal. En somme, la connaissance et l'utilisation des plantes au sein des tribus ethniques sont liées entre autres aux savoirs anciens et au brassage dû aux relations interethniques (Malan et al., 2020).

\section{Conclusion}

Cette étude ethnobotanique a évalué l'importance culturelle des plantes à usage artisanal au sein des tribus Agni du Centre-est et Nord-est de la Côte d'Ivoire. Elle a montré qu'un nombre conséquent de plantes sont utilisées dans l'artisanat. Mais, seuls $14,42 \%$ des plantes ont un indice d'importance culturelle élevé avec 13,46\% d'usage commun des plantes entre ces quatre communautés. De plus, l'évaluation de la concordance des connaissances a montré de fortes différences au niveau des connaissances sur les plantes à usage artisanal entre les quatre tribus. Les savoirs liés aux plantes à usage artisanal s'érodent progressivement, à cause notamment de la désuétude des usages, la non-disponibilité des plantes dut à la dégradation de l'environnement et la concurrence avec les matériaux modernes. La valorisation des connaissances et pratiques ethnobotaniques liées à ces plantes, la sensibilisation des populations sur leurs utilisations rationnelle et leurs prises en compte dans la politique des conservations seraient bénéfiques aux populations.

\section{References:}

1. Gning, O., Sarr, O., Gueye, M., Akpo, L. E. \& Ndiaye, P. M. (2013). Valeur socio-économique de l'arbre en milieu malinké (Khossanto, Sénégal). Journal of Applied Biosciences, 70 : 5617-5631.

2. Hadonou-Yovo, A. G., Houessou, L. G., Lougbegnon, T. O., Adebi, Y., Sinasson, G. K. S., Semevo, D. F., Lange, U. \& Boko, M. (2019). Diversité et formes d'utilisation des espèces ligneuses de la Réserve de biosphère du Mono (Bénin). VertigO, 19 (2) :1-21.

3. Jaccard, P. (1908). Nouvelles recherches sur la distribution florale. Bulletin de la Société Vaudoise des Sciences Naturelles, 44 : 223-270. 
4. Kimpouni, V. \& Nguembo, J. (2018). Diversité floristique et identité culturelle des populations à la périphérie du sanctuaire de Lossi Congo (Brazzaville). Annales de l'Université Marien NGOUABI, 18 (1) : 1734

5. Kouakou, K. A., Barima, Y. S. S., Zanh, G.G., Traoré, K. \& Bogaert, J. (2017). Inventaire et disponibilité des produits forestiers non-ligneux utilisés par les populations riveraines de la Forêt Classée du HautSassandra après la période de conflits armés en Côte d'Ivoire. Tropicultura, 35 (2): 121-136.

6. Kouakou, Y. B., Kougbo, M. D., Konan, A. S., Malan, D. F. \& Bakayoko, A. (2020). Usages traditionnels et disponibilité des plantes exploitées dans l'artisanat chez les populations Koulango et Lobi de la périphérie Est du Parc national de la Comoé. European Scientific Journal, 16 (9) : 295-320.

7. Kouakou, Y. B. (2019). Usages traditionnels des plantes et disponibilité des ressources végétales chez les peuples Koulango et Lobi de la périphérie Est du Parc national de la Comoé. Thèse de Doctorat, Université Nangui Abrogoua (Côte d'Ivoire), p55.

8. Lougbegnon, T. O., Tente, B. A. H., Amontcha, M. \& Codjia, J. T. C. (2011). Importance culturelle et valeur d'usage des ressources végétales de la réserve forestière marécageuse de la vallée de Sitatunga et zones connexes. Bulletin de la Recherche Agronomique du Bénin, $70: 35-46$.

9. Malan, D. F. (2008). Utilisations traditionnelles des plantes et perspective de cogestion des aires protégées de Côte d'Ivoire : Cas du parc national des Iles Ehotile (littoral Est de la Côte d'Ivoire). Thèse de Doctorat, Université Abobo-Adjamé Abidjan, (Côte d'Ivoire), 31 : p57.

10. Malan, D. F., Litta, A. L., Kougbo, M. D., Diop, A. L. \& Kouassi, K. G. (2020). Wild edible plants in four Agni tries of Central-est and Northeast of Côte d'Ivoire : a comparative study. Biodiversitas, 21 (10) : 4896-4902.

11. Ndiaye, I., Camara, B., Ngom, D. \& Sarr, O. (2017). Diversité spécifique et usages ethnobotaniques des ligneux suivant un gradient pluviométrique Nord-Sud dans le bassin arachidier sénégalais. Journal of Applied Biosciences, 113: 11123 - 11137.

12. Ouattara, D., Kouamé, D., Tiébré, M. S., Cisse, A. \& N’guessan, K. E. (2016). Diversité floristique et usages des plantes dans la zone soudanienne du Nord-ouest de la Côte d'Ivoire. Journal of Animal \& Plant Sciences, 31 (1): 4815-4830.

13. Pardo-de-Santayana, M., Tardío, J., Blanco, E., Carvalho, A. M., Lastra, J. J., Miguel, E. S. \& Ramón, M. (2007). Traditional knowledge 
of wild edible plants used in the northwest of the Iberian Peninsula (Spain and Portugal): a comparative study. Journal of Ethnobiology and Ethnomedicine, 3 (27): 1-11.

14. PNUD. (2006). Étude NAMA sur le charbon de bois durable en Côte d'Ivoire. Georgina Wilde, p22.

15. Soro, S., Ouattara, D., Egnankou, W. M. \& Traoré, D. (2014). Usages traditionnels de quelques espèces végétales de la forêt marécageuse classe de Port Gauthier, zone côtière au Sud-ouest de la Cote d'Ivoire. European Scientific Journal, 10 (3): 1857-7881.

16. Tardio, J. \& Pardo-De-Santayana, M. (2008). Cultural importance indices: a comparative analysis based on the useful wild plants of Southern Cantabria (Northern Spain). Economic Botany, 62 : 24 - 39.

17. Tiébré, M. S., Ouattara, D., Vroh Bi, T. A., Gnagbo, A. \& N'Guessan, K. E. (2016). Diversité floristique et disponibilité des plantes utilitaires en zone soudanienne de la Côte d'Ivoire. Journal of Applied Biosciences, 102 : 9699 - 9707.

18. Vroh Bi, T. A., Ouattara, D. \& Kpangui, K. B. (2014). Disponibilité des espèces végétales spontanées à usage traditionnel dans la localité d'Agbaou, Centre-ouest de la Côte d'Ivoire. Journal of Applied Biosciences, 79 : 6386-6396.

19. Yetein, M. H., Tenté, B. H. A. \& Houessou, L. G. (2013). Ethnobotanical study of medicinal plants used for the treatment of malaria in plateau of Allada, Benin (West Africa). Journal of Ethnopharmacology, 146 (1): 154-163.

20. Zeh, C. (2018). "Techniques, formes, Signes et fonctions de la vannerie au Cameroun ». ePhaïstos, 6 (2) :1-31. 